\title{
Ab Initio Theoretical Studies of Atomic and Electronic Structures of III-Nitride (110) Surfaces
}

\author{
H. W. Leite Alves, J. L. A. Alves, \\ Departamento de Ciências Naturais - FUNREI \\ C.P.: 110, CEP: 36300-000, São João del Rei, MG Brazil \\ R. A. Nogueira, \\ Departamento de Física - ICEx - UFMG \\ C.P.: 702, CEP: 30161-970 Belo Horizonte MG Brazil \\ and J. R. Leite \\ LNMS, Departamento de Física dos Materiais e Mecânica - USP \\ C.P.: 66.318, CEP: 05389-970, São Paulo SP Brazil
}

Received February, 1998

\begin{abstract}
We present a systematic theoretical study of several III-nitride (110) surfaces based on accurate, parameter-free, self-consistent total energy and force calculations using the density functional theory, the generalized gradient approximation(GGA) for the exchange-correlation term, and the Full Potential Linear Augmented Plane Wave (FPLAPW) approach associated with the slab supercell model. We studied AlN, BN, GaN and InN and analyzed the theoretical trends for the equilibrium atomic structures and surface band structures. We used supercells built up of 7 atomic layers and a vacuum region equivalent of 5 atomic layers. For the $\mathrm{Ga}$ and $\mathrm{In}$ species, the $3 \mathrm{~d}$ and $4 \mathrm{~d}$ electrons were treated properly as valence electrons. In connection with the atomic structures, we are concerned with the LEED parameters $\Delta_{1 \perp}, \Delta_{1 x}, \Delta_{2} \perp, d_{12} \perp, d_{12 x}$ and $\omega$ for the (110) surface. We analyzed the changes in the bond-lengths and in the bond-angles at the anion and cation sites. We conclude that similarly to the III-arsenide (110) and III-phosphide (110) surfaces, the III-nitride (110) surfaces relax such that the cation-surface atom moves inward and the $\mathrm{N}$-surface atom moves outward. The large Coulomb energy of the III-nitrides as compared with the other III-V compounds reflects in the smaller value of the tilt angle $\omega$ and in the small value of $\Delta_{1} \perp$. To our knowledge, this is the first time the FPLAPW method is used for such a systematic study of III-nitrides, and we compare our results with recent results obtained with other approaches as reported in the literature.
\end{abstract}

\section{Introduction}

The success of crystalline $\mathrm{GaN}$ as an electronic and optical material has motivated enormous theoretical efforts in order to understand the properties of the IIInitrides. Although the III-nitrides crystallize in the hexagonal wurzite phase, with the exception of $\mathrm{BN}$, it is possible to grow epitaxially thin films of $\mathrm{GaN}, \mathrm{InN}$ and $\mathrm{AlN}$ in the zinc-blende structure. In the present work we are concerned with the relaxation of the non-polar (110) surfaces which have had contradicting reports in the literature. We report a systematic study of the atomic and electronic structures of the BN, AlN, GaN and InN (110) surfaces by means of the Full Potential Linearized Augmented Plane Wave(FPLAPW)[1] approach to the Density-Functional Theory(DFT)[2], and compare the results with those obtained by means of other approaches to DFT as reported recently in the literature $[3-6]$.

\section{Theoretical Framework}

In the present work we use accurate, parameter-free, self-consistent total energy and force calculations using the DFT, the generalized gradient approximation(GGA) for the exchange-correlation function[7], and the FPLAPW approach associated to the slab supercell model[8]. We use slabs consisting of seven (110) planes. The lattice constants were fixed at the bulk theoretical equilibrium values, $a_{0}=3.627 \AA$, $4.406 \AA$, 4.554 $\AA, 5.069 \AA$, for $\mathrm{BN}, \mathrm{AlN}, \mathrm{GaN}$ and $\mathrm{InN}$, respectively[9]. We used vacuum regions with a thickness equivalent 
to five atomic layers. This choice should be adequate for minimizing the interaction between contigous surfaces. The central layers of the slab were kept fixed at the bulk positions and the outermost layers of atoms on both sides of the slab were relaxed to geometries given by the calculated total energies and forces. The equilibrium geometry is identified when all forces are smaller than $0.02 \mathrm{eV} / \AA$. This corresponds to a numerical uncertainty of atomic positions of less than 0.05 $\AA$. The muffin-tin radii used were 1.29 a.u., 1.55 a.u., 1.70 a.u. and 1.90 a.u. for the BN, AlN, GaN and InN surfaces, respectively. In each case, the muffin-tin radii were the same for the cation atoms and the nitrogen atoms. The number of the plane waves were determined by $R_{M T-\max } * K_{\max }=8$ in all calculations. With this choice of the cut-off wave vector, the self-consistent total energy converged within $10^{-4} \mathrm{eV}$. The cut-off angular momentum was $\ell=10$ for the wave function inside the spheres and $\ell=4$ for the computation of non-muffin-tin elements. The summation over four Monkhorst-Pack[10] special k-points in the irreducible part of the Brillouin-zone was used to replace Brillouin-zone integrations. The Ga-3d and In-4d electron states were treated explicity as part of the valence band states in order to take into account the hybridizations between Ga-3d and $\mathrm{In}-4 \mathrm{~d}$ and $\mathrm{N}-2 \mathrm{~s}$ states. The parametrization for the GGA approximation was taken from Perdew et al[11].

Table I: Structural parameters for the surface relaxation as defined in Figure 1. The change of the cation-anion distances(labeled $c_{i} a_{j}$ ) between neighbouring atoms in the first layer $(i, j=1$ ) of our slabs is given in the last column.

\begin{tabular}{cllllllll}
\hline \hline & $a_{0}(\AA)$ & $\Delta_{1, \perp}(\AA)$ & $\Delta_{1, x}(\AA)$ & $\Delta_{2, \perp}(\AA)$ & $d_{12, \perp}(\AA)$ & $d_{12, x}(\AA)$ & $\omega\left(^{\circ}\right)$ & $c_{1} a_{1}(\%)$ \\
\hline $\mathrm{BN}$ & 3.627 & 0.215 & 2.967 & 0.043 & 0.994 & 1.639 & 18.08 & -7.17 \\
$\mathrm{AlN}$ & 4.406 & 0.188 & 3.540 & 0.040 & 1.301 & 2.013 & 12.25 & -6.06 \\
$\mathrm{GaN}$ & 4.554 & 0.247 & 3.650 & 0.039 & 1.334 & 2.057 & 15.30 & -5.53 \\
$\mathrm{InN}$ & 5.069 & 0.250 & 4.002 & 0.002 & 1.550 & 2.324 & 13.00 & -4.34 \\
\hline \hline
\end{tabular}

In Table II we show the bond angles obtained in our present calculations for the III-nitrides. The $\alpha$ angle is bigger in the III-nitrides than in the traditional III-V compounds [12], but one still can see that it tends to $90^{\circ}$ along the series and the $\gamma$ angle tends to $120^{\circ}$. These features can be understood if one considers the rehybridization effects being opposed by the repulsion

\section{Results and Discussion}

In Table I we display the structural parameters for the relaxed surfaces as defined in Fig.1. The surface relax in such a way that the cation-atom moves inward tending to a planar threefold coordination with its $\mathrm{N}$-atom neighbours and the uppermost $\mathrm{N}$-atom moves outward into a corresponding pyramidal configuration with its three cation neighbours. It has been claimed that the III-nitride (110) surfaces have anomalous relaxations when compared to the traditional III-V (110) surfaces due to the small tilt angles found in the calculations[6]. However, based on the present and on previous calculations[12,13], we think that the driving mechanism of the relaxation can still be attributed to the early one suggested by Swarts et al.[14] in connection with the GaAs(110) surface, and analyzed in detail for GaAs, GaP, InAs and InP (110) surfaces in [12]: the surface group $\mathrm{V}$ atom changes into a more p-like configuration(the $\alpha$ angle tends to be close to $90^{\circ}$ ) and the surface group III atom changes into a more $s p^{2}$ like configuration with respect to their bulk $s p^{3}$ configuration. In this way the top layer geometry lies between the bulk geometry(tetrahedral bonding with the angles $\alpha=\beta=109.5^{\circ}$ ) and the geometry obtained by putting the cation and the anion in their respective small molecule configurations, e.g. the planar $\mathrm{GaH}_{3}$ molecule with $120^{\circ}$ bond angle and the pyramidal $\mathrm{As}_{3}$ molecule with $92.1^{\circ}$ bond angles, thus close to $90^{\circ}$. 
to compare the behaviour of different compounds. In Table II we compare the trends of the tilt angle $\omega$, the perpendicular displacement $\Delta_{1 \perp}$, the angles $\alpha, \beta$ and $\gamma$ with the trend of the Coulomb energy $\frac{Z e^{2}}{\epsilon a_{0}}$, where $a_{0}$ is the zinc-blende theoretical lattice constant, $\mathrm{Z}$ is the longitudinal effective charge[16], $e$ is the electron's charge, and $\epsilon$ is the dielectric constant. As can be seen from the Table II the various relaxation parameters follow the expected trend of the Coulomb energy. For instance the angle $\alpha$ decreases, getting closer to $90^{\circ}$, when the Coulomb energy decreases. The larger Coulomb energy of the III-nitrides compared to the other III-V compounds reflects in its big value of the $\alpha$ angle, the small value of the tilt angle $\omega$, and in the small value of $\Delta_{1 \perp}$. Similar analysis has been done by Alves et al. in connection to II-VI compounds[13].

Table II: Calculated parameters as defined in Figure 1 and Coulomb energy(CE)(see text).

\begin{tabular}{lcclllr}
\hline \hline & $\omega\left(^{\circ}\right)$ & $\Delta_{1, \perp} / a_{0}(\%)$ & $\alpha\left(^{\circ}\right)$ & $\beta\left(^{\circ}\right)$ & $\gamma\left(\left(^{\circ}\right)\right.$ & $\mathrm{CE}(\mathrm{eV})$ \\
\hline $\mathrm{BN}$ & 18.1 & 5.8 & 99.8 & 115.5 & 123.2 & 0.537 \\
$\mathrm{AlN}$ & 12.3 & 4.3 & 102.2 & 114.4 & 120.7 & 1.267 \\
$\mathrm{GaN}$ & 15.3 & 5.5 & 100.3 & 116.0 & 119.6 & 1.227 \\
$\mathrm{InN}$ & 13.0 & 4.9 & 101.3 & 115.7 & 117.4 & 1.151 \\
\hline \hline
\end{tabular}

Table III: Relaxation energy $(\Delta \mathrm{E})$, surface energy $(\sigma)$, normal displacement anion-cation $\left(\Delta_{\perp}\right)$, layer rotation angle $(\omega)$, and surface bond-length-change $(\Delta b)$.

\begin{tabular}{lllllr}
\hline \hline & BN & AlN & GaN & InN & Reference \\
\hline$\Delta \mathrm{E}(\mathrm{eV} / \mathrm{at})$ & 0.56 & 0.24 & 0.23 & 0.16 & this work \\
& 0.50 & 0.23 & 0.22 & 0.15 & {$[3]$} \\
& - & 0.23 & 0.22 & 0.15 & {$[4]$} \\
$\sigma(\mathrm{eV} / \mathrm{at})$ & 1.61 & 0.23 & & & {$[6]$} \\
& 0.97 & 1.04 & 0.83 & 0.71 & {$[3]$} \\
& - & 1.07 & 0.97 & 0.94 & {$[4]$} \\
& & 1.30 & & & {$[6]$} \\
$\Delta_{\perp}(\AA)$ & 0.21 & 0.19 & 0.25 & 0.25 & this work \\
& 0.19 & 0.17 & 0.23 & 0.24 & {$[3]$} \\
& & & 0.11 & & {$[5]$} \\
$\omega\left({ }^{\circ}\right)$ & & 0.14 & & & {$[6]$} \\
& 18.08 & 12.25 & 15.30 & 13.00 & this work \\
& 15.74 & 11.61 & 14.29 & 13.13 & {$[3]$} \\
& & 11.70 & 14.30 & 14.40 & {$[4]$} \\
& & & 7.46 & & {$[5]$} \\
$\Delta \mathrm{b}(\%)$ & -7.17 & -6.06 & -5.53 & -4.34 & this work \\
& -7.8 & -3.6 & -5.3 & -4.9 & {$[3]$} \\
& & -5.9 & -4.9 & -4.3 & {$[4]$} \\
& & & -5.4 & & {$[5]$} \\
& & -5.7 & & & {$[6]$} \\
\hline \hline
\end{tabular}

In the Table III we compare our results to other calculations based on the local density approximation(LDA) to the DFT and we can see that the overall agreement is very good. However, one should note that our results based on the GGA approximation are larger than those based on the LDA approximation(see Ref. 9) 
a)
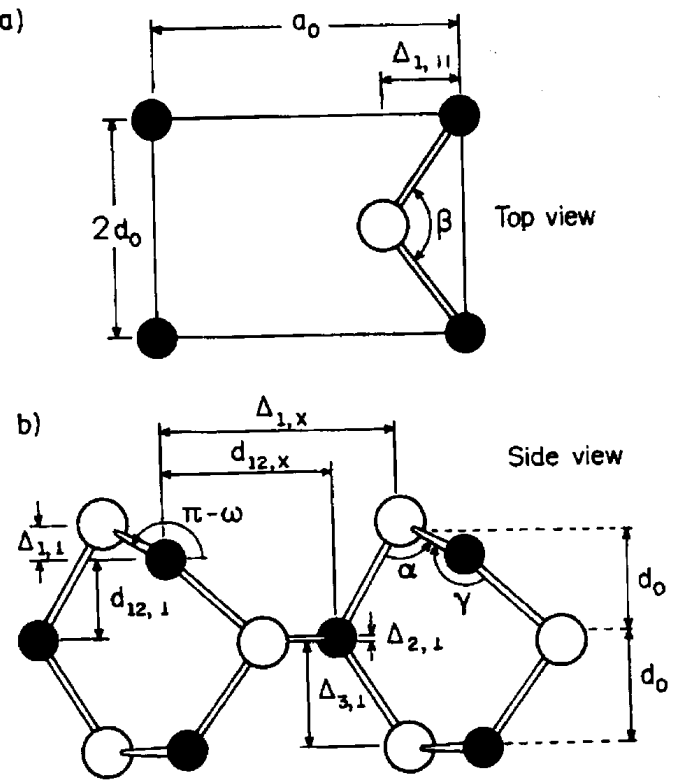

Figure 1. Atomic geometry for III-nitrides (110) surfaces.(a) Top view of the surface unit cell. (b) Side view of the first three layers of the (110) surface. Open circles are anions and the shaded circles are cations. $a_{0}$ is the theoretical bulk lattice constant and $d_{0}=\sqrt{2}\left(a_{0} / 4\right)$.

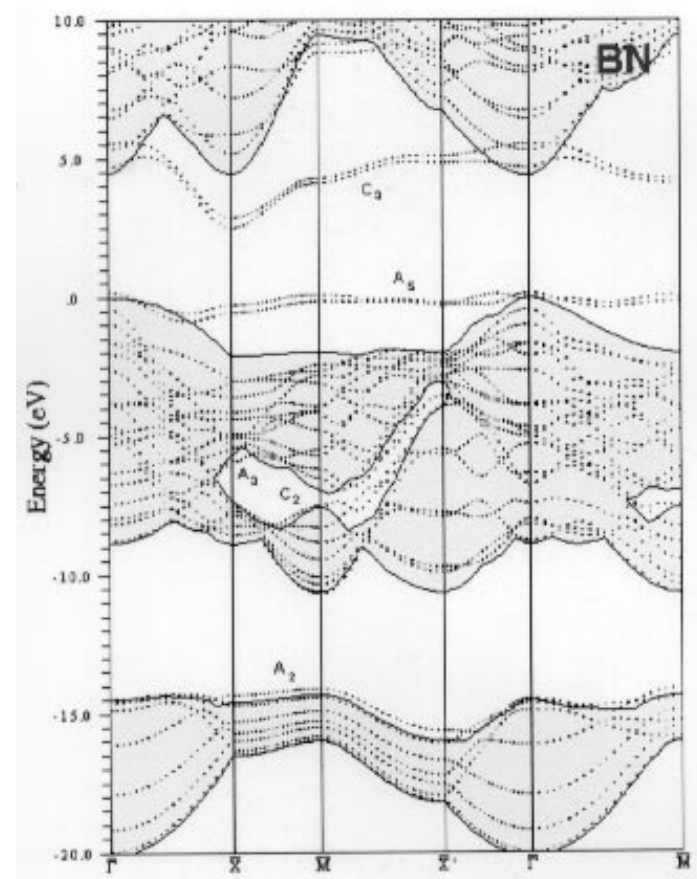

Figure 2. Two-dimensional band structure of the relaxed BN (110) surface. The projected bulk band structure corresponds to the shaded regions. The labels $A_{i}, C_{i}$ refer to surface states localized at surface anions(cations).

The surface band structures of the four compounds are qualitatively similar to those of the III- $\mathrm{V}$ and IIVI compounds[12,13] and are shown in Figs. 2-5. The shaded areas correspond to the projected bulk band structures and the surface states are superimposed.
One can identify the surface states localized at surface anions $\left(A_{i}\right)$ and at surface cations $\left(C_{i}\right)$. The common features to all compounds are as follows. (a) There are surface states in the fundamental gap between the valence band and the conduction band arising from both the empty cation-derived dangling bond $C_{3}$ and the occupied anion-derived dangling bond $A_{5}$ states. For $\mathrm{BN}$ and $\mathrm{AlN}$, the $A_{5}$ anion state has a "concave" dispersion to be compared to the flat dispersion of GaN, InN, GaAs, GaP, InAs, InP(110) surfaces[12]. (b) The surface-state gaps $\Delta E_{s}$, increases in the order $\Delta E_{s}(\bar{X})<\Delta E_{s}(\bar{M}) \leq \Delta E_{s}\left(\bar{X}^{\prime}\right)$. (c) There are surface states $A_{3}$ (p-like) and $C_{2}$ (s-like) lying in the central region of the bulk valence bands("stomach" gap) that show strong dispersion. One can note a constriction of the stomach gap at the $\bar{M}$ for BN and AlN, as compared to the other III-V compounds. (d) There are s-like surface states $A_{2}$ lying in the lower part of bulk valence-band gap. (e) The $3 \mathrm{~d}$ Ga band is shown to lie around $-14.0 \mathrm{eV}$, superimposed to the $2 \mathrm{~s}$ band of $\mathrm{N}$. (f) There are no s-like surface states $C_{1}$ in the heteropolar band gap, around the point $\bar{M}$, in contradistinction to the other III-V compounds(see Ref. 12). Our results have a very good overall agreement with the results reported by Agrawal et al.[5] for the band structure of the GaN (110) surface.

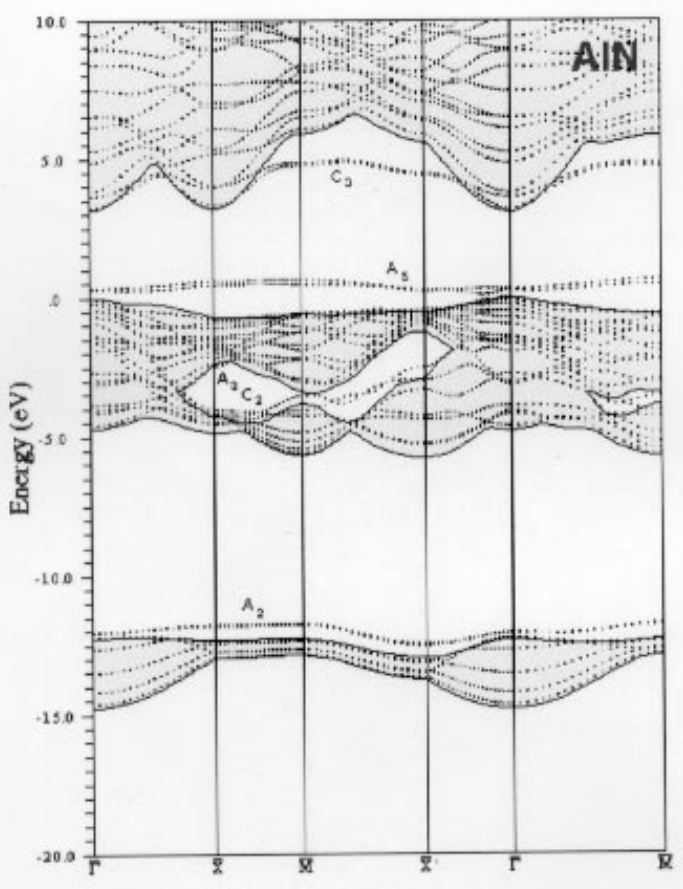

Figure 3. Same as Figure 2 for the relaxed AlN (110) surface. 


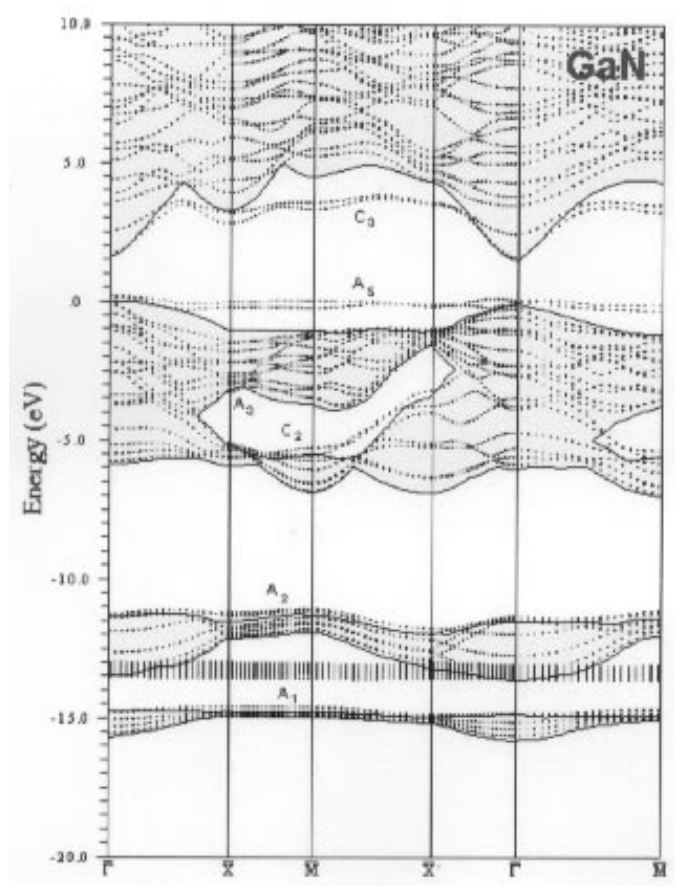

Figure 4. Same as Figure 2 for the relaxed GaN (110) surface.

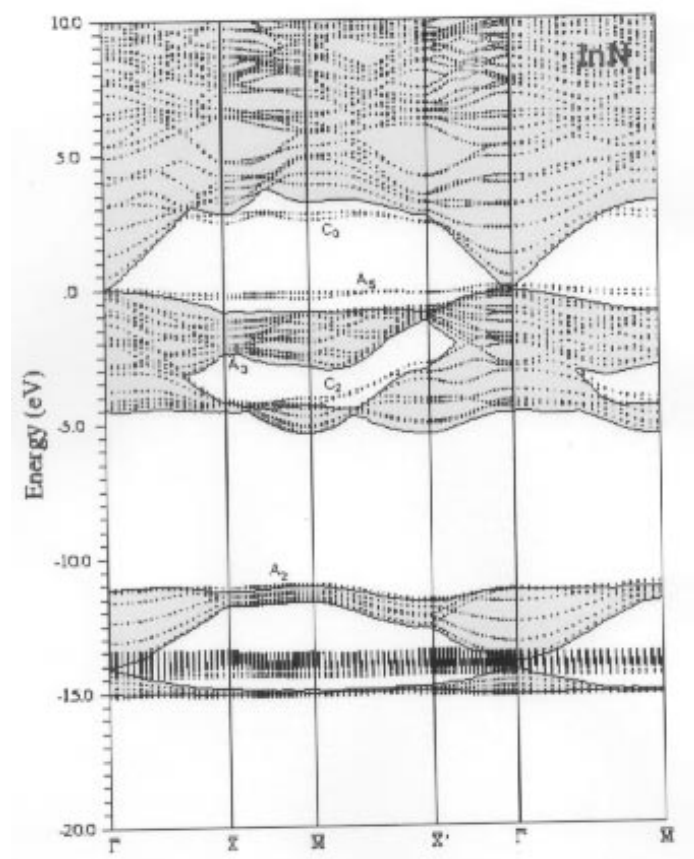

Figure 5. Same as Figure 2 for the relaxed InN (110) surface.

A more detailed analysis of the electronic states and their connection to the atomic structure of the surface is in progress and will come up soon in another publication.

\section{Conclusions}

We have presented well-converged $a b$ initio calculations of the equilibrium atomic and electronic structures for the (110) surfaces of III-nitrides, based on the Full Potential Linear Augmented Plane Wave method. To our knowledge this is the first time that such a systematic calculation has been done using the FPLAPW method and the GGA approximation. The relaxation geometric parameters obtained for the III-nitrides are smaller than those for the other III-V and II-VI compounds. This can be understood as a manifestation of the ionic forces counteracting the covalent forces in the rearrangements at the surfaces, as can be inferred by analyzing the Coulomb energy trend along the series. Still, we think that the "small-molecule" model[14] is a good one to understand the surface rearrangements, if the ionic forces are taken into account.

\section{Acknowledgements}

Work supported by FAPEMIG and CNPq. The computational resources were provided by CENAPAD$\mathrm{MG} / \mathrm{CO}$.

\section{References}

[1] P. Blaha, K. Schwarz, P. Dufek and R. Augustyn, Computer Code Wien97, Technical University of Vienna, 1979.

[2] P. Hohenberg and W. Kohn, Phys. Rev. 136, B864(1964).

[3] U. Grossner, J. Furthmüller and F. Bechstedt, Phys. Rev. B 58, R1722 (1998).

[4] A. Filippetti, V. Fiorentini, G. Cappellini and A. Bosin, phys. stat. sol. (b) 170, 265 (1998).

[5] B. K. Agrawal, P. Srivastava, S. Agrawal, Surf. Sci. 405 , 54 (1998).

[6] R. Pandey, P. Zapol and M. Causà, Phys. Rev. B 55, R16009 (1997).

[7] J. P. Perdew, J. A. Chevary, S. H. Vosko, K. A. Jackson, M. R. Pederson, D. J. Singh and C. Fiolhais, Phys. Rev. B 46, 6671 (1992).

[8] M. Schlüter, J. R. Chelikowsky, S. G. Louie and M. L. Cohen, Phys. Rev. B12, 4200 (1975).

[9] These values are larger than the respective experimental ones, $3.615 \AA, 4.37 \AA, 4.50 \AA$ and $4.98 \AA$, and are in agreement with the calculations by C. Stampfl and C. G. Van de Valle (Phys. Rev. B 59, 5521 (1999)). Our GGA values are to be compared to the values obtained using the local density approximation(LDA) to the DFT which are usually smaller than the experimental values.

[10] H. J. Monkhorst and J. D. Pack, Phys. Rev. B 13, 5188 (1976).

[11] J. P. Perdew, S. Burke and M. Ernzerhof, Phys. Rev. Lett. 77, 3865 (1996).

[12] J. L. A. Alves, J. Hebenstreit and M. Scheffler, Phys. Rev. B 44, 6188 (1991). 
[13] J. L. A. Alves, K. Watari and A. C. Ferraz, Solid State Commun. 87, 1001 (1993).

[14] C. A. Swarts, W. A. Goddard III and T. C. McGill, J. Vac. Sci. Technol. 17, 982 (1980).

[15] R. V. Kasowski, M. -H. Tsai and J. D. Dow, J. Vac. Sci.
Technol. 85, 953 (1987); M. -H. Tsai, R. V. Kasowski and J. D. Dow, Solid State Commun. 64, 231 (1987).

[16] G. Lucovsky, R. M. Martin and E. Burstein, Phys. Rev. B 4, 1367 (1971). 